\title{
NMR Detection Using Laser-Polarized Xenon as a Dipolar Sensor
}

\author{
J. Granwehr ${ }^{\text {a }}$ J.T. Urban ${ }^{\text {a }}$ A.H. Trabesinger ${ }^{\mathrm{a}, 1}$ A. Pines ${ }^{\mathrm{a}, *}$ \\ ${ }^{a}$ Materials Sciences Division, Lawrence Berkeley National Laboratory, and \\ Department of Chemistry, University of California, Berkeley, CA 94720, USA
}

\begin{abstract}
Hyperpolarized ${ }^{129}$ Xe can be used as a sensor to indirectly detect NMR spectra of heteronuclei that are neither covalently bound nor necessarily in direct contact with the Xe atoms, but coupled through long-range intermolecular dipolar couplings. In order to reintroduce long-range dipolar couplings the sample symmetry has to be broken. This can be done either by an asymmetric sample arrangement, or by breaking the symmetry of the spin magnetization with field gradient pulses. Experiments are performed where only a small fraction of the available ${ }^{129}$ Xe magnetization is used for each point, so that a single batch of xenon suffices for the point-by-point acquisition of a heteronuclear NMR spectrum. Examples with ${ }^{1} \mathrm{H}$ as analyte nucleus show that these methods have the potential to obtain spectra with a resolution that is high enough to determine homonuclear $J$ couplings. The applicability of this technique with remote detection is discussed.
\end{abstract}

Key words: NMR, Distant dipolar field, Long-range dipolar couplings, Intermolecular multi-quantum coherences, Indirect detection, Remote detection, Hyperpolarized xenon, Sensitivity PACS: 07.05.Fb, 07.05.Kf, 76.60.Jx

\section{Introduction}

In recent years, it was shown that hyperpolarized xenon is an exquisitely sensitive sensor of its direct environment [1]. Applications of xenon as a probe

* Corresponding author. Fax: 1-510-486-5744.

Email address: pines@cchem.berkeley.edu (A. Pines).

URL: http://waugh.cchem. berkeley.edu (A. Pines).

1 Present address: Physical Chemistry Laboratory, ETH Zürich, 8093 Zürich, Switzerland

Preprint submitted to Elsevier Science 28 February 2005 
typically require it to be either bound to, adsorbed $[2,3]$, or in sufficient proximity $[4,5]$ to the analyte. Alternatively, supramolecular cages can be synthesized with a large affinity to bind Xe, where the specificity for a certain target comes from a ligand that is connected to the cage through a linker [6]. Most of these experiments capitalize on changes of the xenon electron cloud that are reflected in changes in xenon chemical shift and/or relaxation behavior. More specific information can be obtained when xenon is involved in the relaxation of the analyte to be probed [7]. The SPINOE technique yields direct quantitative measures about NMR active nuclei in the (temporary) molecular environment of Xe. However, all the aforementioned applications require either solubility of xenon in the analyte [7] (or the analyte in xenon [8]), affinity of xenon to binding sites [6,9-11], or direct physical interaction $[4,12]$.

Another recent application of hyperpolarized xenon gas is its use as an information carrier in experiments with remote detection of NMR. Remote detection is a technique to separate encoding and detection in an NMR experiment temporally and spatially in order to optimize both steps independently [13]. Information about an analyte is encoded onto the spin magnetization of a mobile carrier, which then is moved to a detector that can read out this magnetization with high sensitivity. Remote detection has been applied successfully to measure the chemical shift of ${ }^{129} \mathrm{Xe}$ on the surface of a porous material, and for imaging at low and at high magnetic fields [14]. However, so far there is no experiment to encode the NMR spectrum of a heteronucleus onto the sensor, especially not if the sensor and the analyte are not in direct contact with each other.

An independent field of research that attracted considerable attention during the last decade involves distant dipolar fields (DDF) in liquids [15] and their application to studies of the physiochemical environment of a molecule. Warren et al. showed that interactions can be established between nuclei with high gyromagnetic ratios $\gamma$, separated on a meso- to macroscopic scale, up to the order of $1 \mathrm{~mm}$ [16]. This allowed them to correlate signals between molecules that were located in separate containers. As dipolar couplings are mediated through space, the only requirement is that the two nuclear species are sufficiently close; they do not need to be coupled or involved in the relaxation of each other. Past studies have almost exclusively been focused on protonated liquids. Only a few groups have explored heteronuclear distant dipolar couplings, mainly towards the end of indirect detection schemes for rare nuclei via their solvent in order to surpass the sensitivity of direct detection [17-21]. A notable attempt to use xenon for indirect detection of $\mathrm{CH}_{4}$ gas in a xenon $/ \mathrm{CH}_{4}$ gas mixture can be found in $[19,22]$.

In this work we combine the high signal intensity of laser-polarized ${ }^{129} \mathrm{Xe}$ and the flexibility in sample composition offered by correlating signal over meso- to macroscopic scales via distant dipolar couplings. We spatially and 
spectrally correlate the signal of gaseous xenon in a sealed sample tube with the liquid surrounding it. The experiments reported here demonstrate the possibility of using hyperpolarized xenon not only as a probe of its direct environment, but extends its applicability beyond the microscopic scale. These experiments can also be seen as a useful complement - in the sense of adding spectral information - to two recently proposed experiments which use dipolar fields for local magnetometry. In [23], ${ }^{1} \mathrm{H}$ and ${ }^{13} \mathrm{C}$ resonances of chloroform dissolved in liquid xenon were used for quantitative measurements of xenon magnetization; in [24] it is proposed to use ${ }^{3} \mathrm{He}$ gas in lung tissue to measure and quantify susceptibility changes in nearby blood vessels upon loading them with paramagnetic contrast agents.

The results of the present study are then used to assess the feasibility of experiments where the encoding of a heteronuclear NMR spectrum onto xenon is combined with remote detection, and potential benefits of such an approach are discussed.

\section{Theory}

The theory of distant dipolar field effects in NMR has been worked out by several groups. The most relevant work with regard to our experiments was done in the Warren group $[20,21,25,26]$. We are summarizing the equations required to understand the characteristic features of the experiments presented in this article. Furthermore the influence of self-diffusion is analyzed, which is a more important issue when working with gases than in the case of liquids, and we are commenting on some sensitivity-related aspects that are important for a possible application of this approach with remote detection, especially the importance of multiplicative noise.

\subsection{Signal in a DDF experiment}

The magnetic field, which is given by

$$
\mathbf{B}=\mu_{0}(\mathbf{H}+\mathbf{M})=\mathbf{B}_{0}+\mathbf{B}_{\mathrm{d}}
$$

depends not only on $\mathbf{H}$, but also on the magnetization density $\mathbf{M}=\sum_{i}\left\langle\underline{\mu}_{i}\right\rangle / V$ of the sample, where the $\left\langle\underline{\mu}_{i}\right\rangle$ are the magnetic moments of the individual spins. $\mathbf{B}_{\mathrm{d}}$ is called the demagnetizing field of the sample. High-field NMR studies in the presence of a large applied magnetic field $\mathbf{B}_{0}=B_{0} \tilde{\mathbf{z}}$ are not sensitive to the entire $\mathbf{B}_{\mathrm{d}}$, only the secular components are retained. We are considering a two-spin system where spin $S=1 / 2$ shall be the analyte spin, 
and spin $I=1 / 2$ is the sensor spin, which is hyperpolarized ${ }^{129} \mathrm{Xe}$ in our case. The magnitude of the total spin magnetization density before any radio frequency (rf) irradiation is

$$
M_{0}=M_{0}^{I}+M_{0}^{S}=\frac{\hbar}{2}\left(N_{I} \gamma_{I} P_{I}+N_{S} \gamma_{S} P_{S}\right)
$$

where $N_{I, S}$ is the number density and $P_{I, S}$ is the spin polarization of the $I, S$ spins, respectively. While $P_{S}$ is given by the Boltzmann law, which is $P_{S}=$ $\left|\gamma_{S}\right| \hbar B_{0} / 2 k T$ within the high temperature approximation, the polarization $P_{I}$ of the ${ }^{129} \mathrm{Xe}$ is determined by the efficiency of the spin-exchange optical pumping process and can exceed 0.5 under optimum conditions [27].

If the sample is spherical and magnetically isotropic, i.e. if the magnetization is uniform throughout the sample, dipolar interactions add up to zero. But to conclude that dipolar interactions are negligible because of the $r^{-3}$ dependence is wrong, because the number of spins at a given distance increases with $r^{2}$. The sum of all dipolar interactions on a given spin only falls off with $r^{-1}$, and longrange dipolar interactions have to be considered whenever the magnetization of the sample is a function of location. By neglecting molecular diffusion (which can cause a redistribution of the sample magnetization), by assuming that the relevant nuclear magnetization $\mathbf{M}(s, t)$ arises from a single line $S$ spectrum and a single line $I$ spectrum, and if the spatial distribution of $\mathbf{M}(s, t)$ is only along $\tilde{\mathbf{z}}$, the equation of motion of magnetization in the absence of an rf excitation can be written as

$$
\begin{aligned}
\frac{\partial}{\partial t} \mathbf{M}(s, t)= & \mathbf{M}(s, t) \times \gamma\left\{\mathbf{B}_{0}+G_{z}(t) s+\mathbf{B}_{\mathrm{d}}(s, t)+\mathbf{B}_{\mathrm{r}}(t)+\mathbf{B}_{\mathrm{n}}(s, t)\right\} \\
& -\mathbf{R}\left(\mathbf{M}(s, t)-\mathbf{M}_{0}\right)
\end{aligned}
$$

where $G_{z}(t)=\partial B_{z} / \partial z$ is a pulsed linear field gradient along $\tilde{\mathbf{z}}, s$ is the position of a magnetization volume element along $\tilde{\mathbf{z}}, \mathbf{R}=\operatorname{diag}\left(T_{2}^{-1}, T_{2}^{-1}, T_{1}^{-1}\right)$ is the relaxation matrix, $\mathbf{B}_{\mathrm{r}}(t)$ is the radiation damping field, which is generated by currents induced in the rf coils by the precessing magnetization, and $\mathbf{B}_{\mathrm{n}}(s, t)$ includes environmental and instrumental magnetic noise and inhomogeneities.

The sequence using gradient pulses to break the symmetry of the sample is shown in Fig. 1. The first gradient pulse leads to a helical distribution of the analyte magnetization along $z$,

$$
M^{S+}\left(t_{1}, s\right)=M_{x}^{S}\left(t_{1}, s\right)+i M_{y}^{S}\left(t_{1}, s\right)=\tilde{M}^{S+}\left(t_{1}\right) \exp \left(-i \gamma_{S} G_{z} T s\right)
$$

where $\tilde{M}^{S+}\left(t_{1}\right)$ is the $S$ magnetization in the absence of a gradient pulse, and $T$ is the length of the gradient pulse. Afterwards, a $\pi / 2$ pulse is applied on $S$, and simultaneously a tipping pulse with flip angle $\beta$ is applied on $I$. The $S$ pulse stores one component of $M^{S+}, e . g . M_{x}^{S}$ in case of a $y$ pulse [28], to $M_{z}^{S}$. 


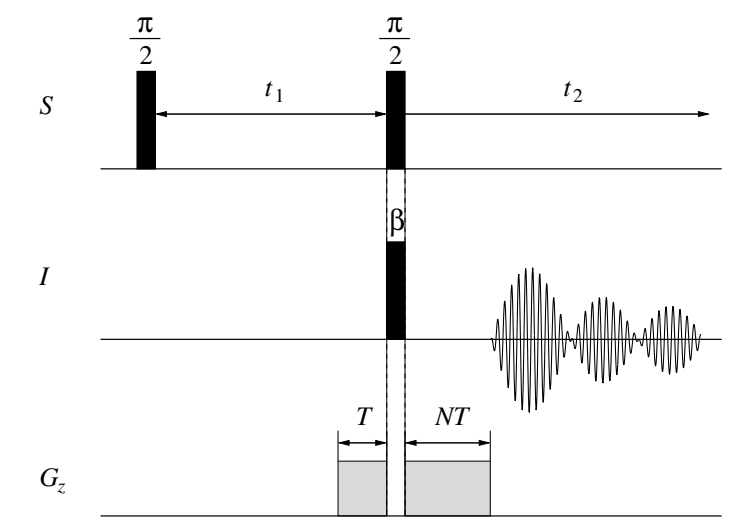

Fig. 1. Pulse sequence of a DDF-detected NMR experiment. $S$ denotes the analyte nucleus of interest. $I$ is the sensor spin - in our case ${ }^{129} \mathrm{Xe}$. A first gradient pulse $G_{z}$ along the axis of the static magnetic field is applied right before the second $\pi / 2$ pulse on $S$, and a second $G_{z}$ pulse follows the pulse on $I$ with a flip angle $\beta \ll \pi / 2$.

This polarization creates a spatially modulated $\mathbf{B}_{\mathrm{d}}(s)$ along $z$, which is acting on the precessing sensor magnetization $M^{I+}$. If the spin density is uniform (e.g. inside an ellipsoidal sample) and the magnetization varies only in a single direction $z$, modulated over distances much smaller than the smallest sample dimensions so that edge effects can be neglected, the secular contribution of the resulting dipolar field seen by a heteronucleus has an effective value of

$$
\mathbf{B}_{\mathrm{d}}(s)=\mu_{0} \Delta_{S} \frac{2 M_{z}(s) \tilde{\mathbf{z}}}{3},
$$

where $\Delta_{S}=\left[3(\tilde{\mathbf{s}} \cdot \tilde{\mathbf{z}})^{2}-1\right] / 2=1$.

If $\mathbf{B}_{\mathrm{d}}(s, t), \mathbf{B}_{\mathrm{r}}(t)$, and $\mathbf{B}_{\mathrm{n}}(s, t)$ are very small compared to $\mathbf{B}_{0}$, it is convenient to use a frame of reference rotating at the angular velocity $\omega_{0}=-\gamma \mathbf{B}_{0}$. At $t_{2}=0$ after the second $\pi / 2$ pulse on the $S$ and the $\beta$ pulse on the $I$ spins, assuming all pulses to be $y$ pulses, the longitudinal and transverse components of the magnetization are

$$
\begin{aligned}
M_{z}^{S}(s) & =M_{0}^{S} \exp \left(-t_{1} / T_{2}^{S}\right) \cos \left(\Delta \omega_{S} t_{1}-\gamma_{S} G_{z} T s\right) \\
M^{I+}\left(0^{+}\right) & =M_{x}^{I}=M_{0}^{I} \sin (\beta)
\end{aligned}
$$

with the resonance offset $\Delta \omega_{S}=\omega_{S}-\omega_{0, S}$, and the transverse relaxation time $T_{2}^{S}$ of the $S$ spins. If $S$ and $I$ spins are in different containers, the spatial distribution of $B_{\mathrm{d}}$ of the $S$ spins as seen by the $I$ spins is not simply given by Eq. (5), but also depends on the sample geometry and the pitch $\lambda=2 \pi /\left(\gamma G_{z} T\right)$ of the magnetization helix. The configuration used for the experiments consists of a sample tube containing the xenon inside a larger tube with the analyte. If $\lambda$ is smaller than the diameter of the inner sample tube, the spatial distribution of $B_{d}$ gets blurred out considerably, leading to a reduced refocusing of the sensor magnetization. The effect of $\lambda$ on the signal is easy to determine 
experimentally. It is also possible to calculate it numerically, but it is difficult to assess analytically. Therefore we will just summarize the theory for a homogeneous mixture of analyte and sensor medium in order to obtain an upper limit for the achievable signal. $\lambda$ shall be small compared to the smallest sample dimensions, so that Eq. (5) is valid, but large enough that we can neglect diffusion. This is usually not a very severe restriction for liquid, but it typically is a major limitation for gases, which will be discussed separately. With this assumption, the sensor magnetization after the second gradient pulse and precession during $t_{2}$ becomes

$$
\begin{aligned}
M^{I+}(s)=M_{0}^{I} & \sin (\beta) \exp \left(-\frac{t_{2}}{T_{2}^{I}}\right) \exp \left[i \left\{\Delta \omega_{I} t_{2}-\gamma_{I} G_{z} N T s\right.\right. \\
& \left.\left.-\frac{2}{3} \gamma_{I} \mu_{0} M_{0}^{S} \exp \left(-\frac{t_{1}}{T_{2}^{S}}-\frac{t_{2}}{T_{1}^{S}}\right) \cos \left(\Delta \omega_{S} t_{1}-\gamma_{S} G_{z} T s\right) t_{2}\right\}\right] .
\end{aligned}
$$

If $T_{1}^{S}$ would be infinite, we could use the Jacobi-Anger expansion

$$
\exp (i x \cos \varphi)=\sum_{k=-\infty}^{\infty} i^{k} \mathrm{~J}_{k}(x) \exp (i k \varphi)
$$

where $\mathrm{J}_{n}$ is the Bessel function of the first kind of order $n$. If we assume for now that $t_{2} \ll T_{1}^{S}$ such that $\exp \left(-t_{2} / T_{1}^{S}\right)$ is approximately constant during $t_{2}$, we can apply the expansion into Bessel functions and then analyze the effect of relaxation in a separate step. Let us therefore substitute $M_{\tau}^{S} \equiv M_{0}^{S} \exp \left(-t_{1} / T_{2}^{S}-t_{2} / T_{1}^{S}\right)$ and $M_{\tau}^{I} \equiv M_{0}^{I} \exp \left(-t_{2} / T_{2}^{I}\right)$, which also helps to simplify the following expressions. The observable $I$ magnetization now becomes

$$
\begin{aligned}
M^{I+}(s)= & M_{\tau}^{I} \sin (\beta) \exp \left(i \Delta \omega_{I} t_{2}\right) \times \\
& \sum_{k=-\infty}^{\infty} i^{k} \mathrm{~J}_{k}\left(-\frac{2}{3} \gamma_{I} \mu_{0} M_{\tau}^{S} t_{2}\right) \exp \left[i\left(k \Delta \omega_{S} t_{1}-\left(k \gamma_{S}+N \gamma_{I}\right) G_{z} T s\right)\right]
\end{aligned}
$$

In order for some of the magnetization to be non-zero after spatial averaging, assuming an integer number of turns of the magnetization helix along the sample axis over the height of the detection coil, one of the terms in Eq. (10) must be constant with respect to position, and therefore must have a net coefficient of zero for $s$. This requires

$$
N=-k \frac{\gamma_{S}}{\gamma_{I}}
$$

for there to be a signal. In our experiments, we usually select $k=-1$, which 
gives

$$
M_{\mathrm{DDF}}^{I+}=i M_{\tau}^{I} \sin (\beta) \exp \left[i\left(\Delta \omega_{I} t_{2}-\Delta \omega_{S} t_{1}\right) \mathrm{J}_{1}\left(-\frac{2}{3} \gamma_{I} \mu_{0} M_{\tau}^{S} t_{2}\right)\right]
$$

For a more intuitive description, one can say that if the $M^{I+}$ magnetization is wound up as a helix along $z$ with the same pitch as the helix of the $S$ spin, i.e. $\gamma_{S} G_{z} T=\gamma_{I} G_{z} N T$, some of the crushed $M^{I+}$ magnetization gets refocused because $B_{d}^{S}(s)$ has the same spatial dependence as $M^{I+}$.

With inductive detection, the signal is proportional to $\partial M_{\mathrm{DDF}}^{I+} / \partial t_{2}$. Since detection is done in the lab frame, one has to convert $M_{\mathrm{DDF}}^{I+}$ into this frame as well, and we get

$$
\begin{aligned}
& \frac{\partial M_{\mathrm{DDF}}^{I+}}{\partial t_{2}}=M_{\tau}^{I} \sin (\beta) \exp i\left(\omega_{I} t_{2}-\Delta \omega_{S} t_{1}\right) \times {\left[\left(\omega_{I}+\frac{i}{t_{2}}\left(1+\frac{t_{2}}{T_{2}^{I}}-\frac{t_{2}}{T_{1}^{S}}\right)\right) \mathrm{J}_{1}\left(\frac{2}{3} \gamma_{I} \mu_{0} M_{\tau}^{S} t_{2}\right)\right.} \\
&\left.-\frac{2 i}{3}\left(1-\frac{t_{2}}{T_{1}^{S}}\right) \gamma_{I} \mu_{0} M_{\tau}^{S} \mathrm{~J}_{0}\left(\frac{2}{3} \gamma_{I} \mu_{0} M_{\tau}^{S} t_{2}\right)\right] \\
& \approx M_{\tau}^{I} \sin (\beta) \omega_{I} \exp \left[i\left(\omega_{I} t_{2}-\Delta \omega_{S} t_{1}\right) \mathrm{J}_{1}\left(\frac{2}{3} \gamma_{I} \mu_{0} M_{\tau}^{S} t_{2}\right)\right]
\end{aligned}
$$

The approximation can be made because at high field the real part of the expression in square brackets, which is scaled with $\omega_{I}$, is several orders of magnitude larger than the imaginary part except for very short $t_{2}$, which are not relevant for the signal in our experiments. In the absence of relaxation, the maximum signal amplitude is not dependent on $M_{0}^{S}$, but only on $M_{0}^{I}$. Since the maximum of $\mathrm{J}_{n}(x)$ is at $x \approx 1.84$, the position of the signal maximum is at

$$
t_{2} \approx \frac{2.75}{\mu_{0} \gamma_{I} M_{0}^{S} \exp \left(-\frac{t_{1}}{T_{2}^{S}}\right)}=2.75 \tau_{d}^{I} \exp \left(\frac{t_{1}}{T_{2}^{S}}\right)
$$

where $\tau_{d}^{I}=\left(\mu_{0} \gamma_{I} M_{0}^{S}\right)^{-1}$ is the characteristic dipolar demagnetizing time of spin $I$ in the presence of the dipolar field of spin $S$ [30].

As a next step, we have to reconsider the above assumption to neglect longitudinal relaxation of $M_{z}^{S}(s)$ during $t_{2}$. Because $M_{z}^{S}$ in the argument of $\mathrm{J}_{1}$ relaxes with $T_{1}^{S}$, one does not get a pure Bessel shaped envelope of the signal, but something like a "stretched" Bessel. The part of $M_{z}^{S}$ that is modulated along $z$, which is responsible for the refocusing of $M^{I+}$, diminishes until it finally reaches its limiting value when $M_{z}^{S}=M_{0}^{S}$. Therefore it is usually not possible to observe the zero-crossing of the Bessel function. If we perform a 
series expansion of $\mathrm{J}_{1}(A x)$, where $A$ is a constant and $x$ is the variable term, we get

$$
\mathrm{J}_{1}(A x)=\frac{1}{2} A x-\frac{1}{16} A^{3} x^{3}+\frac{1}{384} A^{5} x^{5}+O\left(x^{7}\right) .
$$

Usually even with large values of $M_{0}^{S}$ it is still reasonable to only consider the lowest order term of this expression. Since this term is linear in $A$ and $x$, we can simply determine the $t_{2}$ dependence of $M_{\tau}^{S}$ as

$$
\begin{aligned}
M_{\tau}^{S}\left(t_{2}\right) t_{2} & =\int_{0}^{t_{2}} M_{0}^{S} \exp \left(-\frac{t_{1}}{T_{2}^{S}}-\frac{t}{T_{1}^{S}}\right) \mathrm{d} t \\
& =M_{0}^{S} \exp \left(-\frac{t_{1}}{T_{2}^{S}}\right) \frac{T_{1}^{S}}{t_{2}}\left[1-\exp \left(-\frac{t_{2}}{T_{1}^{S}}\right)\right] .
\end{aligned}
$$

If we neglect instrumental constants, the signal becomes

$$
\begin{array}{r}
s\left(t_{2}\right)=\frac{\partial M_{\mathrm{DDF}}^{I+}}{\partial t_{2}}=-\frac{\mu_{0}}{3} \gamma_{I}^{2} B_{0} T_{1}^{S} \sin (\beta) M_{0}^{I} M_{0}^{S}\left[1-\exp \left(-\frac{t_{2}}{T_{1}^{S}}\right)\right] \times \\
\exp \left(i \omega_{I} t_{2}-\frac{t_{2}}{T_{2}^{I}}\right) \exp \left(-i \Delta \omega_{S} t_{1}-\frac{t_{1}}{T_{2}^{S}}\right) .
\end{array}
$$

The signal is bilinear in $M_{0}^{S}$ and $M_{0}^{I}$ as long as experiments are done in the linear regime of $\mathrm{J}_{1}\left(2 t_{2} / 3 \tau_{d}\right)$, which is fulfilled well if $t_{2}<\tau_{d}$. The maximum refocusing of $M^{I+}$ for $t_{2} \gg T_{1}^{S}$ gets delayed by the effect of the longitudinal relaxation on $M_{z}^{S}\left(t_{2}\right)$. The degree of refocusing of the $I$ spins corresponds to the value it would have reached at $t_{2}=T_{1}^{S}$ in the absence of $T_{1}^{S}$ relaxation. On the other hand, as long as $t_{2} \ll T_{1}^{S}$, we get

$$
s\left(t_{2}\right) \approx-\frac{\mu_{0}}{3} \gamma_{I}^{2} B_{0} \sin (\beta) M_{0}^{I} M_{0}^{S} t_{2} \exp \left(i \omega_{I} t_{2}-\frac{t_{2}}{T_{2}^{I}}\right) \exp \left(-i \Delta \omega_{S} t_{1}-\frac{t_{1}}{T_{2}^{S}}\right) .
$$

Therefore as long as experiments are done in the linear regime of $\mathrm{J}_{1}\left(2 t_{2} / 3 \tau_{d}\right)$, the signal is bilinear in the analyte and the sensor magnetization.

With a non-transient detection method where the signal is read out at one particular point of the evolution during $t_{2}$, we get the maximum signal amplitude at $t_{2}=T_{2}^{I}$ as

$$
\left|s^{\max }\right|=\frac{\mu_{0}}{3} \gamma_{I}^{2} B_{0} \sin (\beta) M_{0}^{I} M_{0}^{S} T_{2}^{I} \exp \left(-\frac{t_{1}}{T_{2}^{S}}-1\right)
$$

if $s\left(t_{2}\right)$ is given by Eq. (18). 
So far, only the case has been discussed where the spectrum of the $I$ spin and the spectrum of the $S$ spin consist of one line each. For the sensor spin, this is a realistic assumption, and all the experiments presented here use a single-line sensor. But the $S$ spin can very well have a more complicated NMR spectrum. In this case the trigonometric addition formula $\cos \left(\Delta \omega_{S} t_{1}-\gamma_{S} G_{z} T s=\right.$ $\cos \left(\Delta \omega_{S} t_{1}\right) \cos \left(\gamma_{S} G_{z} T s\right)+\sin \left(\Delta \omega_{S} t_{1}\right) \sin \left(\gamma_{S} G_{z} T s\right)$ can be used, which allows to incorporate the $\cos \left(\Delta \omega_{S} t_{1}\right)$ and the $\sin \left(\Delta \omega_{S} t_{1}\right)$ into the argument of the Bessel function. For $k= \pm 1$, this leads to a linear superposition of the signals from the $S$ spins as long as the experiments are done in the linear regime of the Bessel function. Therefore the time-domain signal along $t_{1}$ matches the evolution of the $S$ spins in a one-pulse NMR spectrum.

An interesting feature of experiments with Xe dissolved in the analyte is that the two magnetization patterns are experiencing the same $\mathbf{B}_{0}$ inhomogeneities. A $B_{0}$ dependent correlation between the $I$ and $S$ spins is obtained for $k$ space spectral components of the $B_{0}$ inhomogeneities smaller than $\lambda^{-1}$, which manifests itself as ridges in the $2 \mathrm{D}$ spectrum with a slope of $-k \gamma_{S} / \gamma_{I}$. The ridges can be straightened by a shearing transform or by a projection of the spectrum onto an axis perpendicular to the tilting axis of the lines [28], and the high-resolution spectrum of the $S$ spin can be restored. With the Xe and the analyte in different containers, such a correlation of inhomogeneities is usually much less effective because $I$ and $S$ spins are not occupying the same space. With a tube in a tube configuration, analyte and sensor only overlap along the $z$ axis, which leaves only $z$ inhomogeneities to be correlated. And since $\lambda$ is typically on the order of the diameter of the inner sample tube, only $B_{0}$ inhomogeneities with low $k$-space spectral components can be correlated, which can be shimmed out reasonably well anyway.

If the recycle delay time $t_{r}$ between consecutive traces (i.e. between the detection pulse of one trace and the preparation pulse of the next trace) is on the order of $T_{1}^{S}$ or shorter such that a considerable amount of modulated $M_{z}^{S}(s)$ does not relax, some of the $S$ magnetization can evolve into double or zero quantum coherences. This can cause spectral artifacts along $t_{1}$, which either diminish with a time constant $T_{1}^{S}$ or with the diffusion time constant of the analyte helix along $z$, whichever is shorter. A strong spoiler gradient, which is usually applied to dephase leftover $M^{+}$magnetization after the acquisition, does not improve the situation because longitudinal magnetization is not affected by a gradient pulse. To avoid these artifacts, it is either necessary to wait several $T_{1}^{S}$ between subsequent traces, to apply simultaneously a spoiler gradient and an $\mathrm{rf}$ saturation pulse on the $S$ spins, or to design a phase cycle that considers coherences that build up over more than one transient [31]. 


\subsection{Influence of radiation damping}

The precession of a highly magnetized spin species induces an oscillating current in the receiver coil which is strong enough that the coil itself produces a transverse resonant magnetic field that causes a torque on the spins. This radiation damping can be quantitatively described as a modification to the Bloch equations [32]. The angle $\beta$ between $\mathbf{M}(t)$ and the equilibrium magnetization $\mathbf{M}_{0}$ changes as

$$
\frac{\mathrm{d} \beta}{\mathrm{d} t}=-\frac{\sin (\beta(t))}{\tau_{\mathrm{r}}},
$$

with the characteristic rate of radiation damping $\tau_{\mathrm{r}}^{-1}=2 \pi Q \eta|\gamma||\mathbf{M}(t)|$, where $Q$ is the quality factor and $\eta$ the filling factor of the $\mathrm{rf}$ coil. Usual strategies to prevent radiation damping involve detuning or overcoupling of the rf circuit of the probe, modifying the circuit to suppress radiation damping, or, if possible, apply only small tipping angle rf pulses. In our experimental scheme, another possibility to diminish radiation damping is to apply a gradient pulse immediately after the rf pulse, which averages out the integrated magnetization over the whole sample volume. However, recent studies have shown that the combination of radiation damping and the presence of a DDF from an abundant solvent nucleus at a high magnetic field can lead to turbulent spin dynamics [26] even if magnetization has been crushed directly after an rf pulse. This fact by itself would complicate DDF detection of NMR, even though the influence of instrumental and environmental magnetic noise has not been included in these studies. Fortunately, since in a heteronuclear experiment the encoding circuit is not used for detection, we are able to reduce its $Q$ and detune it without losing sensitivity, as long as we still can apply pulses short enough to obtain the desired pulse bandwidth on the analyte spins. And considering the results in [33], it is advisable to apply gradient pulses as late in the sequence as possible, because any turbulence effects add up during the course of an experiment. On the detection side, radiation damping is of minor importance because the pulse tipping angles are small, leading to only a small transverse component of $\mathbf{M}$. Therefore radiation damping will not be considered further in the following discussion.

\subsection{Influence of multiplicative noise}

Fluctuations of the environment of a sample that influence the propagator of a spin system lead to an error of the spin magnetization, which is the signal inducing quantity of an NMR experiment [34]. This noise is multiplicative. Fluctuations of $\mathbf{B}_{0}$, which is denoted as $\mathbf{B}_{n}$ in Eq. (3), is typically the dominant source of multiplicative noise (or $t_{1}$ noise) in liquid-state NMR experiments [35]. Because $\left|\mathbf{B}_{n}\right| \ll\left|\mathbf{B}_{0}\right|$, the components of $\mathbf{B}_{n}$ that are not 
parallel to $\mathbf{B}_{0}$ get truncated, and $\mathbf{B}_{n} \approx B_{n, z} \equiv B_{n}$. Even though $B_{n}$ could be spatially inhomogeneous, it is spectrally homogeneous. All spins see the same environmental fluctuations, thus each signal component gets convoluted with the same noise contribution. Spin magnetization precessing in the transverse plane accumulates an additional phase contribution

$$
M^{\prime}(t)=M^{+}(t) \exp \left(-i \gamma \int_{0}^{t} B_{n}\left(t^{\prime}\right) \mathrm{d} t^{\prime}\right) \approx M^{+}(t)\left[1-\gamma B_{n} t\right]
$$

where $M^{\prime}(t)$ is the transverse spin magnetization affected by multiplicative noise, and $M^{+}(t)$ is the noiseless quantity. The correlation time of $B_{n}$ is low typically on the order of $t_{2}^{\max }$. Therefore during a transient evolution of magnetization, $B_{n}$ can be approximated as being constant. This noise is affecting the $S$ spins during $t_{1}$ and the $I$ spins during $t_{2}$. The phase error on the $S$ spins gets converted into an amplitude error by the storage pulse. If we assume that $B_{n}$ during $t_{1}$ is not correlated with $B_{n}$ during $t_{2}$, which might be somewhat oversimplified but is often a practical assumption, the two noise terms add in quadrature. The standard deviation $\sigma_{M}$ of the multiplicative noise in the transient dimension can then be approximated by

$$
\sigma_{M}\left(t_{2}\right) \approx \sqrt{\left(\gamma_{S} k \sigma_{B} t_{1}\right)^{2}+\left(\gamma_{I} \sigma_{B} t_{2}\right)^{2}}\left|s\left(t_{2}\right)\right|
$$

where $\sigma_{B}$ is the standard deviation of $B_{n}$. According to Eq. (17) the signal $s\left(t_{2}\right)$ in a DDF experiment is a product of a rising component and a decaying component; it reaches its maximum at $t_{2}=T_{2}^{I}$. This causes an amplification of the impact of multiplicative noise compared to a free induction decay (FID), which depends on $T_{2}^{I}, t_{2}^{\max }$, and the relative contribution of $\left(\gamma_{S} k \sigma_{B} t_{1}\right)^{2}$ to $\left(\gamma_{I} \sigma_{B} t_{2}\right)^{2}$. But with transient detection, the on-resonant signal-to-noise ratio (SNR) due to multiplicative noise in a DDF experiment does not exceed the SNR of an FID experiment with the same $t_{2}^{\max }$ and no apodization by more than a factor two. Only if $t_{2}^{\max }$ in an FID experiment can be kept short compared to $t_{2}^{\max }$ of a DDF experiment, this figure increases.

With single point detection at $t_{2}=T_{2}^{I}$, the multiplicative noise contribution can be considerable especially with a sensor spin with a long $T_{2}^{I}$ like ${ }^{129} \mathrm{Xe}$. It might appear that the low $\gamma$ of ${ }^{129} \mathrm{Xe}$ compared to, say, protons is an advantage, but since the refocusing of the magnetization with a high $\gamma$ sensor is faster, there is no net advantage of the low $\gamma_{I}$ in this experiment. Multiplicative noise that adds during $t_{1}$ causes mainly problems when high spectral resolution is required with correspondingly long $t_{1}$. If $k$ in Eq. (11) is not equal to \pm 1 , the noise can also increase substantially, again depending on $t_{1}$.

Because the spectrum of the analyte is recorded point-by-point with basically no correlation of multiplicative noise between adjacent data points, its sensitivity is stronger affected by multiplicative noise than it would be the case with 
direct detection of an FID, where each line simply gets convoluted with the same noise pattern that has a spectral density narrower than a typical line. Therefore only the reproducibility of an FID detected spectrum is affected, which is important for example for the efficiency of a phase cycle. The contribution of multiplicative noise can be estimated by comparing the noise level along the direct dimension of the spectrum with the noise level along the indirect dimension. Multiplicative noise only eventually limits the maximum SNR, but it does not limit the minimum detectable analyte concentration. However, the noise caused by an intensive line can cover weak lines of the same analyte spectrum. Correspondingly, if the direct FID of ${ }^{129} \mathrm{Xe}$ is not fully dephased by the second gradient pulse, the remaining portion also contributes to the multiplicative noise, even if it is subtracted by a phase cycle.

Because the main contribution to multiplicative noise usually are fluctuations of $B_{0}$, using a frequency lock improves $\sigma_{M}$ considerably. Except for analytes with only a single NMR line it was usually very difficult to obtain a spectrum at all without frequency lock in our experiments.

If the analyte spectrum consists of weak and strong lines that are well separated from each other and their positions are approximately known, one possibility to recover the full sensitivity for the weak lines is to apply semiselective pulses that do not excite the strong lines. Oversampling along the indirect dimension also improves the SNR [36], but this improvement is, at least for white noise, only proportional to the square root of the number of points and thus the detection time, and is therefore equivalent to signal averaging, except that it can be adjusted in finer steps.

Because the achievable SNR is eventually limited by multiplicative noise anyway, $\beta$ can be reduced until the additive SNR is about equal to the multiplicative SNR. Smaller $\beta$ allow to apply more pulses until all of the Xe polarization of a single batch is used up, which in turn improves the SNR.

\subsection{Magnetization decay and diffusional mixing of xenon gas}

During a batch mode experiment, the polarization of ${ }^{129} \mathrm{Xe}$ gets reduced, because the equilibrium polarization is orders of magnitude lower than the initial polarization. If all the xenon gas was in the active volume $V_{C}$ of the transmitter coil, its longitudinal magnetization before the $(n+1)$ th pulse would be

$$
M_{z}^{I}\left(t_{n+1}^{-}\right)=M_{z}^{I}\left(t_{n}^{-}\right) \cos (\beta) \exp \left(-\frac{t_{r}+n \Delta t_{1}}{T_{1}^{I}}\right) .
$$

The evolution time of the analyte increases by $\Delta t_{1}$ between consecutive transients. Since $t_{n+1}=n t_{r}+n(n+1) \Delta t_{1} / 2$, Eq. (23) can be written as 


$$
\begin{aligned}
M_{z}^{I}\left(t_{n+1}^{-}\right) & =M_{0}^{I} \cos (\beta)^{n} \exp \left(-\frac{n t_{r}+n(n+1) \Delta t_{1} / 2}{T_{1}^{I}}\right) \\
& \approx M_{0}^{I} \exp \left[-n\left(\frac{\beta^{2}}{2}+\frac{t_{r}}{T_{1}^{I}}\right)\right]
\end{aligned}
$$

The approximation is valid for $\beta \ll 1$ and $t_{r} \gg n \Delta t_{1}$. With constant flip angles $\beta$, the signal along the indirect dimension gets reduced in correlation to the drop of the magnetization. This acts like an apodization along $t_{1}$ and causes the line of the analyte to be broadened independently of the relaxation time, but dependent on $\Delta t_{1}$ and on $\beta$. The shorter $\Delta t_{1}$, the broader the line in the indirect dimension of the spectrum becomes. The signal along $t_{1}$ decays as $\left|s\left(t_{1}\right)\right|=s_{0} \exp \left(-t_{1} / \tau_{1}\right)$ with the lifetime

$$
\tau_{1} \approx\left[\frac{1}{T_{2}^{S}}+\frac{t_{r}}{T_{1}^{I} \Delta t_{1}}+\frac{\beta^{2}}{2 \Delta t_{1}}\right]^{-1}
$$

where the same approximations as for Eq. (25) were made. If the decay curve is known such a line broadening can be corrected by either deconvoluting the spectrum or, equivalently, multiplying each time trace with the inverse of the decay function of $M_{z}^{I}\left(t_{n}\right)$. However, such a resolution enhancement generally reduces the sensitivity. Because at least for concentrated analytes the sensitivity at the beginning of the decay along $t_{1}$ is dominated by multiplicative noise, the apodization can be chosen that the noise is about equal to the additive noise. In a next step an apodization for sensitivity enhancement like a matched filter can be applied. For example, a Lorentz-to-Gauss transform seems a very promising filter for this kind of data.

A better solution to avoid sensitivity loss and line broadening would be to determine a variable flip angle scheme [37] that leads to a constant signal during the experiment. The calculation of such a variable flip angle is considerably complicated by the fact that in the sample arrangement with the Xe gas in an inner tube and the analyte in an outer tube, $V_{C}$ contains only a fraction of the total Xe volume. During the course of an experiment, the Xe inside and outside $V_{C}$ are mixed by diffusion, which increases the apparent ${ }^{129} \mathrm{Xe}$ lifetime beyond what would be expected from the setting of $\beta$. The parameters that determine this decay include $\beta, t_{r}$, the self-diffusion constant $D_{\text {Xe }}$ of the xenon gas, which itself depends on the pressure $p$ of xenon (and possibly present inert gases), and the dimensions of the Xe container. A recursive relation can be obtained by assuming a sample tube that extends to infinity in one or in both directions, and that $t_{r}$ is long enough to level out the magnetization of the Xe gas inside $V_{C}$ between subsequent traces of the experiment [38]. Experimentally it was found that the depletion of $M_{z}^{I}\left(t_{n}\right)$ can be fitted reasonably well with a stretched exponential,

$$
M_{z}^{I}\left(t_{n+1}^{-}\right) \approx M_{0}^{I} \exp \left(-\frac{t_{1}^{\alpha}}{\tilde{\tau}_{1}}\right)
$$


where $\tilde{\tau}_{1}$ is the measured lifetime of the signal, and the exponent $\alpha<1$ is responsible for a decay slower than monoexponential, especially for large $n$, since the more the xenon polarization gets depleted in the direct vicinity of $V_{C}$, the more important becomes diffusional mixing of small quantities of xenon gas over longer distances inside the sample tube.

\subsection{Drifts of the xenon sensor resonance frequency}

In combination with the decay of the xenon magnetization, DDF experiments are very sensitive to drifts of the ${ }^{129} \mathrm{Xe}$ resonance frequency between different transients along the indirect dimension [39]. This requires special measures especially because of the large pressure and temperature dependence of the ${ }^{129} \mathrm{Xe}$ chemical shift, which was found to be [40]

$$
\sigma=\sigma_{0}+\sigma_{1} \rho
$$

where $\rho$ is the xenon density in amagat, and $\sigma_{1}=-0.548 \mathrm{ppm} /$ amagat at $25^{\circ} \mathrm{C}$. Assuming an ideal gas, this leads to

$$
\Delta \sigma \approx\left[-1.84 \times 10^{-3} \frac{\mathrm{ppm}}{\mathrm{K}}\right] \frac{p \Delta T}{p_{0}}=\left[-0.0216 \frac{\mathrm{Hz}}{\mathrm{TK}}\right] \frac{p \Delta T B_{0}}{p_{0}} .
$$

This drift can be especially significant for experiments with pure xenon gas as sensor because of the low thermal conductivity of xenon of $56.9 \mu \mathrm{W} \mathrm{cm}{ }^{-1} \mathrm{~K}^{-1}$ at $25^{\circ} \mathrm{C}$ and a pressure of $1 \mathrm{~atm}$ [41], which causes a very slow adaption of the xenon temperature to the temperature of its environment.

If pure xenon gas is used at a pressure of several bar, another reason for a shift of the ${ }^{129} \mathrm{Xe}$ resonance frequency could be the change of the self-demagnetizing field caused by the decreasing magnetization of hyperpolarized ${ }^{129} \mathrm{Xe}$ as sensor during an experiment. This effect would be considerable especially if liquid [23] or supercritical [8] xenon were used. If the xenon and the analyte are mixed, both experience the same demagnetizing field of the xenon, and the frequency shift can be corrected with a frequency lock. In the tube in a tube setup, however, the self-demagnetizing field of the Xe does not get corrected with the lock, but it gets even amplified because its direction is opposite to the direction of the demagnetizing field that the analyte sees.

\section{Experimental Details}

The experiments were performed on a Unity Inova spectrometer (Varian Inc., Palo Alto CA) with a $7 \mathrm{~T}$ widebore magnet (Oxford Instruments, Cambridge 
UK), corresponding to a proton frequency of $300 \mathrm{MHz}$. For the experiments with the Xe diluted inside the analyte a two channel $5 \mathrm{~mm}$ probe (Varian Inc.) was used (broadband channel inner, ${ }^{1} \mathrm{H} /{ }^{19} \mathrm{~F}$ channel outer coil), which was equipped with a separate deuterium lock channel and a $z$ gradient coil. The experiments were done by mixing the analyte and the Xe gas in a $5 \mathrm{~mm}$ outer diameter J-Young tube (Wilmad Labglass, Buena NJ). A 10 mm probe (Varian Inc.) with two channels (a broadband channel using the inner coil, and a ${ }^{1} \mathrm{H} /{ }^{19} \mathrm{~F}$ channel using the outer coil), a separate deuterium channel for frequency locking, and a $z$ gradient coil was used for the experiments with Xe and analyte in different containers.

In principle there are no general restrictions regarding the geometry of the containers with the analyte and the Xe. We put them in two coaxial NMR glass tubes with different diameters. The outer tube was a thick-walled $10 \mathrm{~mm}$ NMR sample tube (Wilmad) that contained the analyte. For Xe a 5 mm outer diameter J-Young tube with a wall thickness of $0.9 \mathrm{~mm}$ was used. It was stabilized inside the $10 \mathrm{~mm}$ tube with a plastic spacer (Fig. 2). The inside of

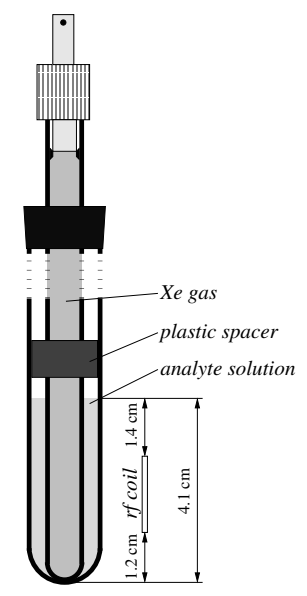

Fig. 2. Setup for the tube in a tube experiment. The analyte was in the outer tube, the sensor in the inner tube. The diameters of the inner and the outer tube were $5 \mathrm{~mm}$ and $10 \mathrm{~mm}$, respectively. The wall thickness of the inner tube was $0.9 \mathrm{~mm}$.

the J-Young tube was coated with SurfaSil (Pierce, Rockford IL) to increase the $T_{1}$ of ${ }^{129} \mathrm{Xe}$. Inside the $7 \mathrm{~T}$ magnet, $T_{1}\left({ }^{129} \mathrm{Xe}\right) \approx 50 \mathrm{~min}$ was measured. The ratio of the Xe volume in the sensitive region of the coil compared to the total tube volume was about $1 / 13$.

For spin-exchange optical pumping, a gas mixture of $1 \%$ naturally abundant Xe, $10 \% \mathrm{~N}_{2}$, and 89\% He (Spectra Gases Inc., Vista CA) was used, which was done using a XenoSpin polarizer (Amersham Health, Durham NC). After polarization, the Xe was condensed into a cold finger immersed in liquid nitrogen. That way the inert gases were separated, and the experiments could be done with pure Xe gas. During thawing the gas was transfered into the J-Young tube. Immediately after removing the liquid nitrogen container, the 
cold finger with the frozen xenon was immersed in hot water $\left(\sim 80^{\circ} \mathrm{C}\right)$ to accelerate thawing and therefore minimizing the depolarization of ${ }^{129} \mathrm{Xe}$. The Xe pressure in the sample tube was $3-5$ bar, and the ${ }^{129} \mathrm{Xe}$ spin polarization was about $7 \%$.

The pulse sequence is shown in Fig. 1. Since Xe cannot be refreshed during an experiment, pulses on ${ }^{129} \mathrm{Xe}$ with only small flip angles $\beta$ had to be used to allow for a reasonable number of points in the analyte spectrum, which was recorded point-by-point. The length of the gradient pulses was fixed at $1 \mathrm{~ms}$, and the gradient amplitude was modified for the different experiments. At the end of the detection period a strong crusher gradient was applied to dephase remaining transverse magnetization. The field gradients do not just serve to break the symmetry of the sample, but also act as coherence pathway filters if they are strong enough [42]. However, with the tube in a tube setup only weak gradients can be applied. The direct FID is only partially suppressed, and only incomplete frequency discrimination is achieved in the indirect dimension. Therefore the phase cycle shown in table 1 was used.

Table 1

\begin{tabular}{|c|c|c|c|}
\hline$S$ Pulse 1 & $S$ Pulse 2 & $I$ Pulse & Receiver \\
\hline $0^{\circ}$ & $0^{\circ}$ & $0^{\circ}$ & $0^{\circ}$ \\
$0^{\circ}$ & $180^{\circ}$ & $180^{\circ}$ & $0^{\circ}$ \\
$0^{\circ}$ & $90^{\circ}$ & $90^{\circ}$ & $0^{\circ}$ \\
$0^{\circ}$ & $270^{\circ}$ & $270^{\circ}$ & $0^{\circ}$ \\
\hline
\end{tabular}

Phase cycle used for the tube-in-a-tube experiments. The first two steps remove the direct FID, and the second two steps select one coherence pathway to get frequency discrimination along $\omega_{1}$.

To simplify the calibration of the setup, a model system was used in addition to the xenon experiments where hyperpolarized ${ }^{129} \mathrm{Xe}$ is replaced by ${ }^{31} \mathrm{P}$ as the sensor nucleus - in particular, phosphoric acid (Fisher Scientific, Fair Lawn, NJ) has proved to be a sensible choice. $\gamma\left({ }^{31} \mathrm{P}\right)$ is only about $50 \%$ higher than $\gamma\left({ }^{129} \mathrm{Xe}\right)$, and both sensors have an NMR spectrum with only one single narrow line. This has the advantage that for quantifying the sensitivity, we do not have to consider all the additional imponderabilities of hyperpolarized gas like not perfectly reproducible polarization of ${ }^{129} \mathrm{Xe}$ between batches, or the continuous depletion and diffusion of Xe magnetization during the course of an experiment. 


\section{Sensitivity Optimization}

\subsection{Gradient calibrations}

An important parameter to optimize the sensitivity of DDF experiments is the gradient strength for maximum signal. A calibration experiment was done with phosphoric acid in the inner and benzene (Fisher Scientific) in the outer tube. The $\mathrm{H}_{3} \mathrm{PO}_{4}$ was mixed with $\mathrm{D}_{2} \mathrm{O}(99 \%$ D, Aldrich Chemical Company, Milwaukee, WI) at a ratio of $\mathrm{H}_{3} \mathrm{PO}_{4}: \mathrm{D}_{2} \mathrm{O}=1: 2$, where the deuterium was used for frequency locking. In these experiments, ${ }^{1} \mathrm{H}$ was the analyte nucleus, and ${ }^{31} \mathrm{P}$ was used as sensor. Figure 3 shows the signal and noise as a function of the gradient strength for different delay times $t_{r}$ between subsequent traces along $t_{1}$. The gradient strength is given in relative units, where $G_{0}$ corresponds to a gradient that dephases the magnetization by one period over the diameter of the inner sample tube. In Fig. $3 \mathrm{a}, t_{r}$ is shorter than $T_{1}$ of ${ }^{31} \mathrm{P}$, and in Fig.

(a)
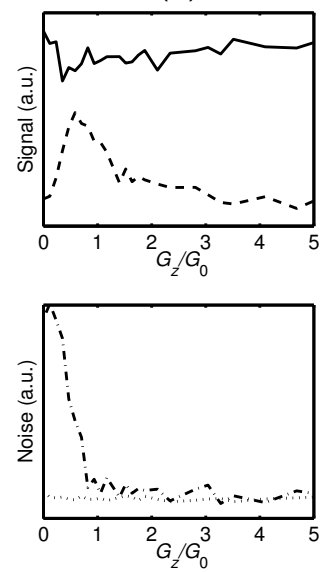

(b)
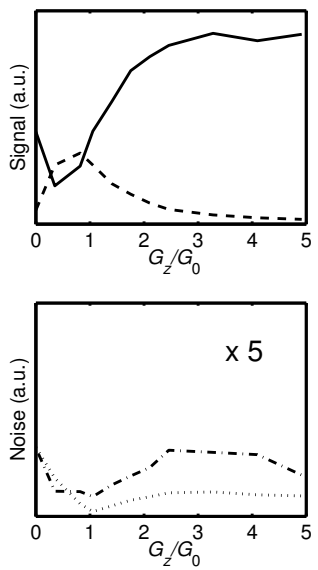

Fig. 3. Signal and noise of a DDF detected ${ }^{1} \mathrm{H}$ NMR spectrum of benzene as a function of the magnetization modulation wavelength using a separated ${ }^{31} \mathrm{P}$ sensor in a coaxial tube geometry. A four-step phase cycle was employed to suppress the direct ${ }^{31} \mathrm{P}$ FID and to ensure frequency discrimination in the indirect dimension. The ratio of the two gradient pulses was fixed to the value $N=\kappa=-\gamma_{H 1} / \gamma_{P 31}=-2.47$. 64 points along the indirect dimension were taken. $G_{z} / G_{0}=d / \lambda$ denotes the strength of a $z$-gradient pulse, relative to a gradient pulse $G_{0}=2 \pi /|\gamma T d|$ that would modulate the same nucleus by one wavelength over the diameter $d$ of the inner sample tube $(d=0.5 \mathrm{~cm})$. A $4 \mathrm{~ms}, 22 \mathrm{G} / \mathrm{cm} z$-gradient spoiler pulse preceded each delay between experiments. (a) $t_{r}=2 \mathrm{~s}$ delay time between experiments. $(b) t_{r}=6 \mathrm{~s}$ between experiments. (upper) Signal as a function of gradient strength. Data points of the ${ }^{1} \mathrm{H}$ signal due to $\mathrm{H}_{3} \mathrm{PO}_{4}$ and $\mathrm{C}_{6} \mathrm{H}_{6}$ are connected by a solid and a dashed line, respectively. (lower) Noise as a function of gradient strength. (dotted line) Average noise level at frequencies with no signal and $\Delta \omega \neq 0$. (dash-dotted line) Noise level at $\Delta \omega=0$. 
$3 \mathrm{~b}, t_{r}$ is about $2 T_{1}\left({ }^{31} \mathrm{P}\right)$. The signal of the ${ }^{1} \mathrm{H}$ line of phosphoric acid as a function of $G_{z}$ is completely different in these two cases. The benzene signal shows the same qualitative behavior in both cases, but the fact that the signal decays to a lower value for the second case leads to the assumption that not only the signal, but also the noise level is considerably different in these two experiments. Figure 3c and d show the noise level at some arbitrary frequency and at $\Delta \omega=0$, where no signal is expected with the particular choice of the reference frequency $\omega_{0}$. The scales in the upper and the lower graphs of Fig. 3a are identical, while the lower graph of Fig. 3b is scaled up by a factor five compared to the upper. If $t_{r}$ is short compared to $T_{1}\left({ }^{31} \mathrm{P}\right)$, noise can be inherited between different shots of the experiment [35]. This mechanism might be one reason for the higher noise amplitude in Fig. 3a compared to Fig. 3b, especially in combination with either radiation damping or interaction with the transverse dipolar field of other ${ }^{31} \mathrm{P}$ nuclei, which acts much like a weak pulse or radiation damping [20]. The phase cycle to remove unwanted coherences leaves a signal at $\Delta \omega_{S}=0$ of considerable intensity in Fig. 3a, because it was not designed to remove coherence pathways of magnetization evolving longer than just during one trace [31]. This is also a reason for the much higher signal with low $G_{z}$ at $\Delta \omega_{S}=0$ from the same nuclei in Fig. 3a than in $3 \mathrm{~b}$. If the direct FID of ${ }^{31} \mathrm{P}$ is incompletely suppressed with a field gradient and the reason is not systematic, it causes an increased noise level over the whole frequency range along $t_{1}$. Since this noise source is independent of the signal induced by intermolecular multi-quantum transitions, it does not appear to be multiplicative. Nevertheless, the noise level along the indirect dimension is considerably higher than along the direct dimension, which is indicative for $t_{1}$ noise.

Another important parameter to describe the sensitivity of DDF experiments with a tube in a tube configuration is the geometrical factor or filling factor, which can be defined as the ratio of the field seen by the analyte versus the field it would see in a mixture of infinite dilution with the sensor. To analytically determine this ratio is very difficult and can be done with satisfying accuracy only for situations that are not really relevant for viable setups. But an experimental determination is straightforward and can be done with the data from the previous paragraph. $\mathrm{H}_{3} \mathrm{PO}_{4}$ (85 weight- $\%$ in $\mathrm{H}_{2} \mathrm{O}$ ) has a total proton density of $72.4 \mathrm{~mol}{ }^{1} \mathrm{H} / \mathrm{L}$, while benzene has $67.6 \mathrm{~mol} / \mathrm{L}$ protons. Both spectra show only a single line, phosphoric acid because of fast proton exchange with water, and benzene because the protons are equivalent. As the phosphoric acid was diluted with $\mathrm{D}_{2} \mathrm{O}$, its effective proton density is about $24 \mathrm{~mol} / \mathrm{L}$. If the signal is taken at the maximum of the benzene peak, an optimum geometrical factor of about 0.35 is obtained for the setup described above. However, as can be seen best from Fig. 3b, the geometrical factor strongly depends on $G_{z}$ and rapidly decays towards a very small value for $\lambda$ shorter than the diameter of the inner tube. 
The above discussion shows the difficulty to compare the sensitivity between DDF experiments and transient one-dimensional (1D) NMR experiments, because the two methods not only show a different signal behavior, but they are also limited by different noise mechanisms. Finally, in both cases the additive, i.e. signal independent, noise from the detector represents the ultimate limit. But with indirect detection, considerably more noise sources have to be considered, making an optimum choice of parameters noticeably more difficult.

\subsection{Multiplicative noise}

Multiplicative noise is caused by low-frequency noise that gets convoluted with the signal. Therefore each noise source affects all of the spins in the same way, thus each spin gets convoluted with the same noise pattern, and the noise has the same characteristics as the signal on which it is superimposed. That multiplicative noise is an important sensitivity limiting factor is shown in Fig. 4, where a ${ }^{19} \mathrm{~F}$ NMR experiment of 2,2,2-trifluoroethanol (99+\%, Aldrich) with ${ }^{31} \mathrm{P}$ of phosphoric acid as sensor was performed. No frequency locking

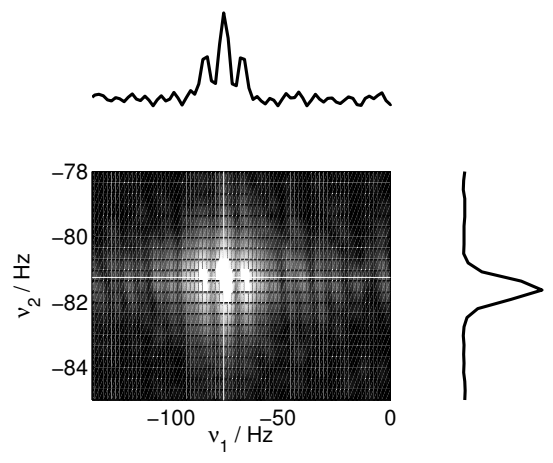

Fig. 4. DDF-detected ${ }^{19} \mathrm{~F}$ NMR spectrum of $\mathrm{CF}_{3} \mathrm{CH}_{2} \mathrm{OH}$ at $7 \mathrm{~T}$, detected with ${ }^{31} \mathrm{P}$ $\left(85 \% \mathrm{H}_{3} \mathrm{PO}_{4}\right)$ as sensor. The phosphoric acid was kept in a tube inside the analyte tube. $\nu_{1}$ is the offest from the ${ }^{19} \mathrm{~F}$ carrier, and $\nu_{2}$ is the offest from the ${ }^{31} \mathrm{P}$ carrier. The white lines show the position of the traces through the spectrum shown above and beneath the $2 \mathrm{D}$ plot.

was employed. A two step phase cycle was used to eliminate the direct FID, and the recycle delay was set to $6.5 \mathrm{~s}$. One can see that the noise shows ridges parallel to the main feature of the ${ }^{19} \mathrm{~F}$ triplet, while the noise intensity is parallel to $\nu_{1}$, which is characteristic for multiplicative noise. The dominance of multiplicative noise along the indirect dimension can also be seen by comparing the noise level of the two traces through the spectrum in Fig. 4.

If a spectrum in the indirect dimension consists of more than one line, multiplicative noise from the individual lines adds in quadrature. Therefore applying selective or semiselective pulses to excite only selected transitions reduces multiplicative noise, especially of weak lines. Figure 5 shows a ${ }^{1} \mathrm{H}$ NMR spectrum 
of a 3:2:1 mixture of isopropanol:phosphoric acid (85\%): $\mathrm{D}_{2} \mathrm{O}$. A DDF-detected

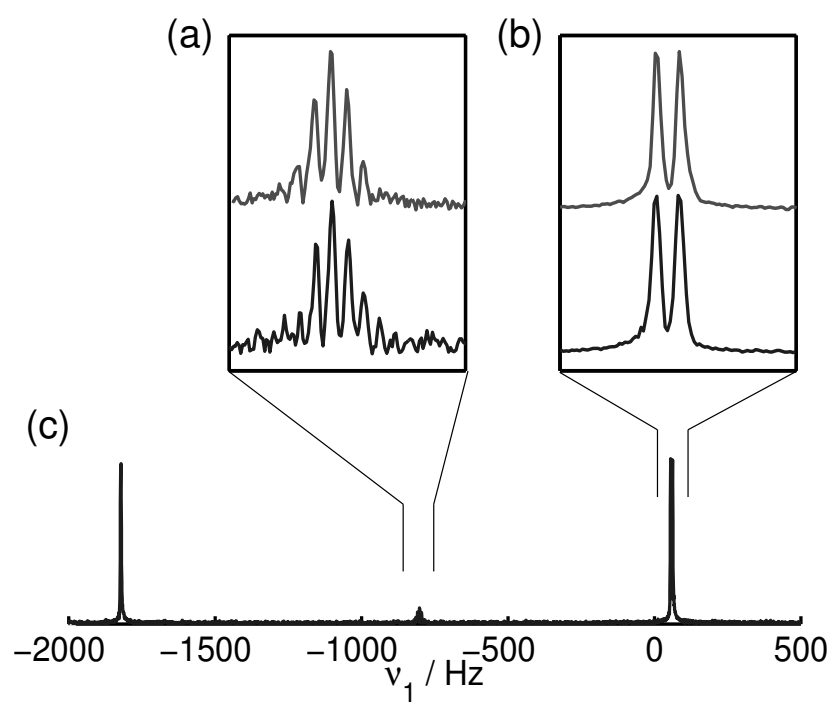

Fig. 5. DDF-detected ${ }^{1} \mathrm{H}$ NMR spectrum of isopropyl alcohol at $300 \mathrm{MHz}$, detected with ${ }^{31} \mathrm{P}$ as sensor. The sample was a 3:2:1 mixture of isopropanol: $\mathrm{H}_{3} \mathrm{PO}_{4}$ (85\%): $\mathrm{D}_{2} \mathrm{O}$. (a) Comparison of the sensitivity of the septet with selective (upper) and non-selective (lower) excitation. (b) Comparison of the sensitivity of the doublet with selective (upper) and non-selective (lower) excitation. (c) Full proton spectrum with non-selective pulses.

spectrum with ${ }^{31} \mathrm{P}$ as sensor was recorded with non-selective pulses and compared to spectra with selective pulses, where only a certain group of lines was excited at a time. The excitation pulse on the analyte was Gauss shaped with a width of $8.2 \mathrm{~ms}$, the storage pulse was a $3 \mathrm{~ms}$ rectangular pulse. While the sensitivity of the methyl protons was not improved considerably with selective excitation, the SNR of the septet could be significantly improved with selective pulses.

\section{Results}

\subsection{Xe in solution}

For batch mode experiments, $\mathrm{Xe}$ is brought into the gas phase above the analyte, and the sample is shaken for about $10 \mathrm{~s}$ prior to the beginning of the experiment to ensure that $\mathrm{Xe}$ is saturated in solution. As an example, Fig. 6 shows a ${ }^{1} \mathrm{H}$ NMR spectrum of deuterated ethyl alcohol $\left(\mathrm{C}_{2} \mathrm{H}_{5} \mathrm{OD}\right.$, Aldrich $)$. The Xe pressure in the gas phase inside the sample tube was 4.6 bar. 256 points were taken along $t_{1}$. Because $T_{1}$ of ${ }^{129} \mathrm{Xe}$ in ethanol is about $160 \mathrm{~s}$ [43], it was necessary to set $t_{r}=1 \mathrm{~s}$, which is less than $T_{1}$ of $\mathrm{C}_{2} \mathrm{H}_{5} \mathrm{OD}$. The total acquisition time was $5 \mathrm{~min}$. The pulse flip angle on the ${ }^{129} \mathrm{Xe}$ was adjusted 
(a)

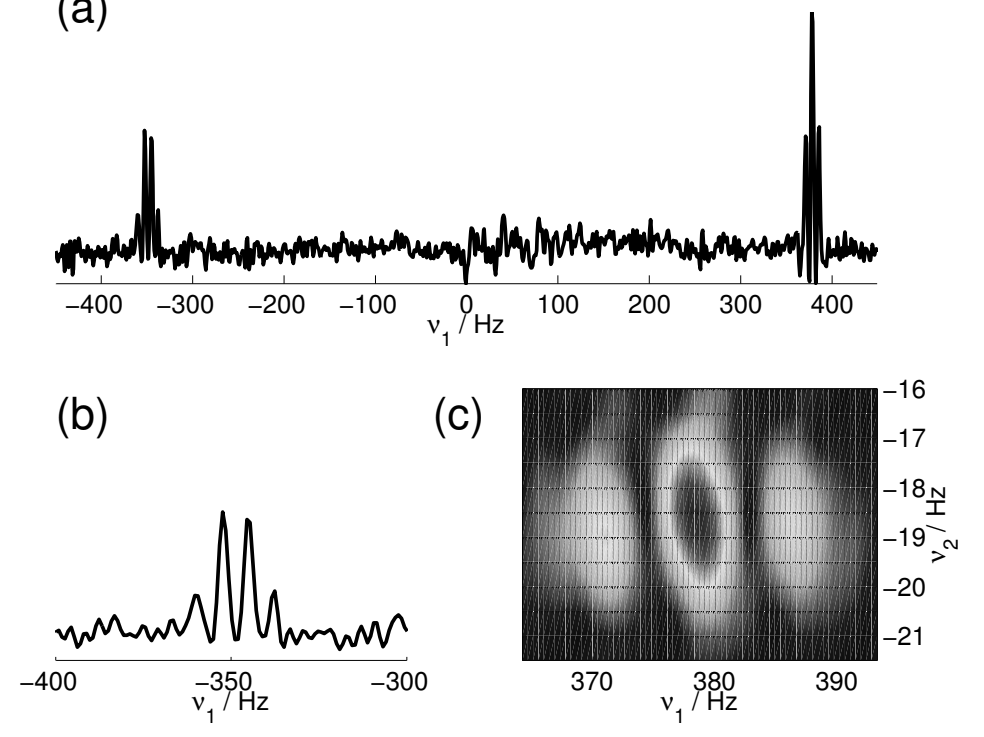

Fig. 6. DDF-detected ${ }^{1} \mathrm{H}$ NMR spectrum of deuterated ethyl alcohol $\left(\mathrm{C}_{2} \mathrm{H}_{5} \mathrm{OD}\right)$ at $7 \mathrm{~T}$, detected through ${ }^{129} \mathrm{Xe}$ as a sensor, which was mixed with the analyte. (a) Full ${ }^{1} \mathrm{H}$ NMR spectrum. (b) Enlargement of the quartet signal from the $\mathrm{CH}_{2}$ group. (c) Pseudocolor plot of the triplet from the $\mathrm{CH}_{3}$ group. $\nu_{1}$ is the indirect dimension, showing the ${ }^{1} \mathrm{H}$ spectrum, and $\nu_{2}$ represents the direct dimension, which is the ${ }^{129} \mathrm{Xe}$ NMR spectrum.

carefully such that the noise level along the direct dimension, which is mainly due to thermal noise in the detection circuit, matches the multiplicative noise along the indirect dimension. In that way, the number of traces could be maximized, giving an optimum overall SNR. The first gradient pulse had an amplitude of $6.1 \mathrm{G} / \mathrm{cm}$, and the second one was set to $22 \mathrm{G} / \mathrm{cm}$. This caused a helix pitch of $\lambda \approx 0.39 \mathrm{~mm}$, which was enough to suppress the direct FID and select one coherence pathway of the $I$ spin magnetization. Therefore no phase cycle was applied. This also helped to reduce multiplicative noise, because the direct FID was dephased and did not contribute. On the other hand, multiplicative noise was increased because some of it was inherited between subsequent traces due to the short $t_{r}$ that did not allow all of the analyte magnetization to relax [35]. Additionally, $t_{r}<T_{1}^{S}$ also caused weak zeroquantum coherences to be visible close to $\nu_{1}=0$.

Figure 6b,c show scale ups of parts of the spectrum containing the lines. Figure $6 c$ shows a pseudocolor plot of the full 2D data after Fourier transform. One can see how inhomogeneities of the analyte and the sensor are correlated, leading to tilted, stretched lines instead of circular features.

With one batch of hyperpolarized xenon, several independent experiments could be performed by reshaking the sample, such that fresh hyperpolarized Xe gas got dissolved in the analyte. In the case of $\mathrm{C}_{2} \mathrm{H}_{5} \mathrm{OD}$, where one experiment was short compared to the $T_{1}$ of ${ }^{129} \mathrm{Xe}$ in the gas phase, a second experiment 
with only a small decrease of sensitivity could be done immediately after the first one.

\subsection{Tube in a tube experiments}

Figure 7 shows a DDF-detected ${ }^{1} \mathrm{H}$ NMR spectrum of water in a field of $7 \mathrm{~T}$, detected with hyperpolarized ${ }^{129} \mathrm{Xe}$. A four step phase cycle was applied, and

(a)

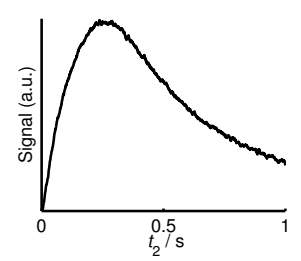

(b)

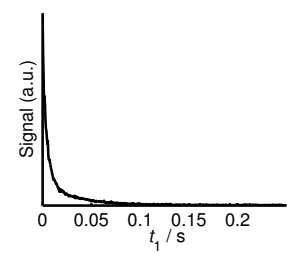

(c)

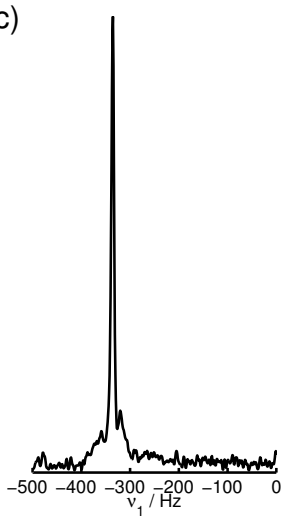

Fig. 7. DDF-detected spectrum of $\mathrm{H}_{2} \mathrm{O}$ at $300 \mathrm{MHz}$, detected with ${ }^{129} \mathrm{Xe}$ as sensor. The Xe was kept in a tube inside the analyte tube. (a) Average signal amplitude along the direct dimension. The signal is a product of a rising component due to the effect of the DDF and a decaying component caused by transversal relaxation of ${ }^{129}$ Xe. (b) Signal decay along the indirect dimension. Because of the depletion of the ${ }^{129}$ Xe magnetization, the signal decays faster than $T_{2}$ of $\mathrm{H}_{2} \mathrm{O}$. (c) ${ }^{1} \mathrm{H}$ NMR spectrum after apodization with the inverse of the signal decay and an exponential decay to remove the arising noise at long $t_{1}$ values.

the recycle delay was set to $3 \mathrm{~s}$. No frequency lock was used. In this experiment, the polarization of ${ }^{129} \mathrm{Xe}$ was only about $1 \%$, and the pressure of the Xe gas was about 2 bar. The pulses on the ${ }^{129}$ Xe had a flip angle of about $\pi / 8$. Figure 7 a shows the absolute value of the signal along the direct dimension, and Fig. $7 \mathrm{~b}$ shows the signal decay along the indirect dimension. The data along the indirect dimension was analyzed by fitting Eq. (27). The product of the inverse of this function with an exponential decay with time constant $\tau=24 \mathrm{~ms}$ was used to apodize the data along $t_{1}$ before the Fourier transform.

Another experiment was done using p-ethoxybenzaldehyde (p-EBA, 99\%, Aldrich). 25 vol.- $\% \mathrm{~d}_{5}$-pyridine (99+\%, Wilmad) was admixed for frequency locking. The xenon pressure was 2 bar. The pulses on the ${ }^{129} \mathrm{Xe}$ had a flip angle of $\beta \approx 4^{\circ}$, which was determined in a separate experiment as being a reasonable choice to get a multiplicative noise level on the same order as the additive noise. The first gradient pulse had an amplitude of $0.078 \mathrm{G} / \mathrm{cm}$, and the second one was $-0.284 \mathrm{G} / \mathrm{cm}$. These gradients were not sufficient to se- 
lect one coherence pathway, so in addition a two-step phase cycle was used to suppress the direct FID of ${ }^{129} \mathrm{Xe}$, consisting of the first two pulses in Tab. 1. But no frequency discrimination was done, so a mirror spectrum is obtained at the negative frequency. The spectral width was chosen big enough to have all NMR lines in one halve of the spectrum. Figure $8 \mathrm{a}$ shows the data in a
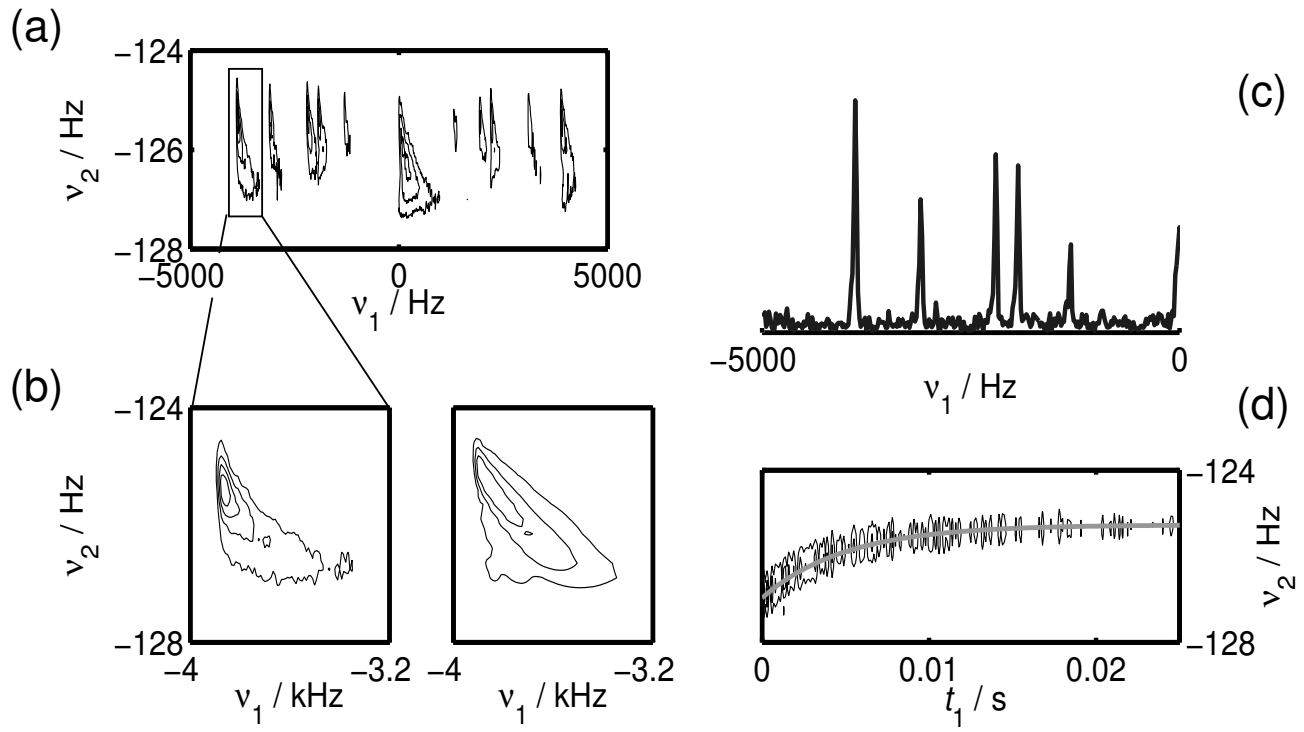

Fig. 8. DDF-detected ${ }^{1} \mathrm{H}$ NMR spectrum of p-ethoxybenzaldehyde at $300 \mathrm{MHz}$, detected with ${ }^{129} \mathrm{Xe}$ as sensor. (a) Contour plot of the full spectrum. (b) Close up of one line (left) and a simulation of the lineshape (right). For this simulation a drift $\Delta \omega_{I}=2 \mathrm{~Hz}$ was assumed. (c) ${ }^{1} \mathrm{H}$ spectrum after correcting for the drift of the ${ }^{129} \mathrm{Xe}$ resonance frequency. (d) Resonance frequency of ${ }^{129} \mathrm{Xe}$ as a function of $t_{1}$. Only a Fourier transform along the direct dimension was performed, and the absolute value of the resulting spectrum was plotted. The gray line corresponds to a fit of the resonance frequency drift, which was used to obtain the spectrum in (c).

$2 \mathrm{D}$ representation of the analyte vs. the sensor frequency without apodizing the signal along $t_{1}$ prior to the Fourier transform. This experiment illustrates well the problems mentioned in section 2. The experimental procedure was optimized to minimize the time between thawing of the xenon and the start of the data acquisition. Because of the hot water used for the thawing, at the beginning of the experiment the xenon gas was several degrees warmer than the ambient temperature, causing a drift of the ${ }^{129} \mathrm{Xe}$ resonance frequency of about $1.7 \mathrm{~Hz}$. This drift, which is shown in Fig. 8d, and the accelerated apparent relaxation due to the diminishing ${ }^{129} \mathrm{Xe}$ polarization cause an asymmetric line. Figure $8 \mathrm{~b}$ compares the experimental lineshape with a simulation using Eqs. (17) and (27). The lineshapes agree qualitatively, which shows that the model presented in section 2 is adequate to describe the observed signal. The exact lineshape in this experiment depends on the temperature evolution and the corresponding $t_{1}$ dependence of the ${ }^{129} \mathrm{Xe}$ chemical shift. On the other hand, the frequency shift caused by the self-dipolar field of ${ }^{129} \mathrm{Xe}$ should be smaller than $0.1 \mathrm{~Hz}$ at this pressure. One can see in Fig. 8a that the lines are 
only symmetric with respect to $\omega_{1}=0$ at frequencies of $\omega_{2}$ where the temperature stabilized (Fig. 8c). During the drift of $\omega_{I}$, the lines on both sides of the spectrum shift in the same direction, which could also be reproduced by the simulation. Figure $8 \mathrm{c}$ shows a spectrum where the effect of the drift of $\omega_{2}$ was corrected numerically prior to Fourier transform along the indirect dimension. All the available data points were used, yet it was possible to retain a linewidth that was given by the spectral resolution and an exponentially decaying apodization function with a time constant of $50 \mathrm{~ms}$, after the signal was first multiplied with the inverse function of the signal decay.

\subsection{Tip mode experiments}

In this class of experiments, a signal builds up because of the asymmetry of the sample and the Xe container. In our experiments with a tube of xenon gas inside the tube with the analyte, this leaves several options like not pushing the tubes all the way down in the probe so they cannot be considered infinitely long, by having the inner tube only half way inside the excitation coil, or by having an analyte that is a multi-phase mixture. In these experiments the direct FID of the ${ }^{129} \mathrm{Xe}$ is suppressed with the same phase cycle as was used to remove the residual FID of the gradient experiment (Tab. 1). Only a two-step cycle was applied, and the spectral width was chosen broad enough that the NMR spectrum fits in half of the bandwidth along $\omega_{1}$. This helps to distinguish signals from artifacts at or near $\Delta \omega_{S}=0$. Figure 9 shows a spectrum of a twophase mixture of water $/ \mathrm{D}_{2} \mathrm{O}(1: 1)$ and methyl acetate (Fisher Scientific). The phase boundary between them was approximately in the center of the coils.

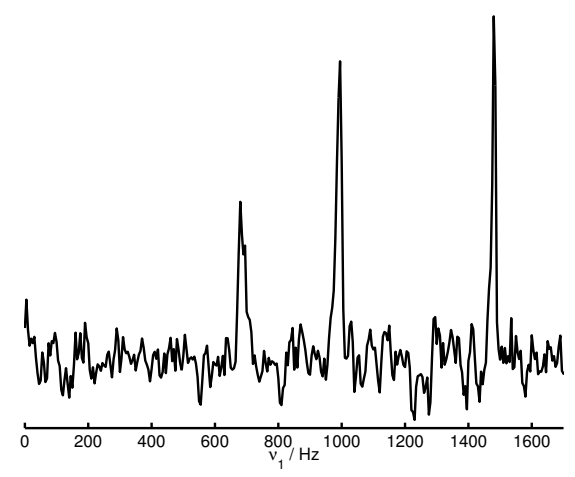

Fig. 9. DDF-detected ${ }^{1} \mathrm{H}$ NMR spectrum of a two-phase mixture of water and methyl acetate at $300 \mathrm{MHz} . \nu_{1}$ is the offset from the ${ }^{1} \mathrm{H}$ carrier. 


\section{Discussion}

We have demonstrated that heteronuclear intermolecular multiquantum coherences can be used to detect the NMR spectrum of an analyte through ${ }^{129} \mathrm{Xe}$, which can be mixed with the analyte or in a different container. In "gradient mode" the interaction distance between a pair of xenon and proton spins can be experimentally set via the gradient strength [16]. However, the effect of washing out the xenon signal due to fast diffusion in between the two gradient pulses has to be considered. Furthermore, with the tube in a tube setup $\lambda$ must be on the order of the diameter of the inner tube or longer to avoid averaging out of the helical $B_{\mathrm{d}}$ distribution that Xe sees from the analyte. In "tip mode" the spatial origin of the detected signal reflects the homogeneity of the magnetic field produced by the protons around the xenon tube.

Keeping the xenon and the analyte in different containers has the advantage of being cleaner to use. It is possible to analyze potentially dangerous or highly sensitive substances while keeping them in a closed environment. Furthermore, the experimental conditions are less dependent on the substance to be analyzed. To mix analyte and sensor has the advantage that the correlation distance of analyte and Xe can be kept small by reducing $\lambda$, which allows to recover homogeneous linewidths by shearing of the spectrum. Additionally, the sensor feels the full demagnetizing field of the analyte. For separate analyte and sensor, a geometrical factor has to be determined that is equivalent to a filling factor in conventional experiments; it depends on the geometry of the setup and the magnetic susceptibility gradients between the involved materials like the container walls, which also makes the tube in a tube setup more difficult to shim. On the other hand, for a mixture the maximum sensitivity depends on the solubility of the analyte in the sensor.

The resolution that can be obtained with DDF encoding is comparable to high resolution spectra with direct detection. Homonuclear $J$ couplings can be resolved in a DDF detected spectrum also with a tube in a tube setup, as can be seen in Fig. 4. But the depletion of the magnetization causes an artificial broadening of the line in the indirect dimension, which does not depend on the inherent linewidth of the analyte. Apodization can correct for that to a certain extent, but at the expense of a reduced sensitivity. A variable flip angle scheme to obtain a constant signal would improve the lineshape and also the sensitivity. However, the simple equations for variable flip angles that do not account for diffusional mixing [37] are unsatisfactory, at least for tube in a tube experiments.

The small angle $\beta$ was one of the sensitivity limiting factor of these experiments. For the Xe in solution experiments another sensitivity limiting factor 
was the low solubility in Xe in polar analytes. The gas exchange between the gas phase and the liquid was too slow to get considerable refreshing of Xe in solution on the time scale of the experiment. Furthermore doping the analyte with a paramagnetic relaxation agent is not possible because such a dopant would reduce the $T_{1}$ of ${ }^{129} \mathrm{Xe}$ as well. For the tube-in-a-tube experiments, on the other hand, a substantial reserve volume of gas outside the sensitive volume of the coil was available, and the continuous refreshing of gas allowed for higher flip angles. Additionally the total experiment time could be optimized by doping the analyte with a paramagnetic salt. The problem here was the low geometrical factor that reduced the DDF of the analyte seen by the sensor.

The examples shown in this report were all done in batch mode. A different possibility would be to use a stopped flow mode where the Xe is replaced between different shots of the experiment. The continuously replaced magnetization allows for large pulse angle excitation and ideally makes the same initial magnetization available for each $t_{1}$ increment. This would also allow to record an unrestricted number of data points for the spectrum of the heteronucleus. And even though the magnetization of the gas is lower in flow mode than in batch mode as co-added inert gases are also circulated without preceding purification, the signal in both modi can be about comparable, since all the available magnetization can be used up in each experiment in stopped flow mode. In batch mode there are no polarization fluctuation due to slow changes of the optical pumping environment, which might be a problem in flow mode. However, preliminary experiments in stopped flow mode did not show any results. This could be explained by an increased self-diffusion of the xenon gas, since the same gas mixture containing $89 \% \mathrm{He}$ and $10 \% \mathrm{~N}_{2}$ that was used for optical pumping was also used for detection. If during detection the gas diffuses over a distance larger than the helix pitch created during encoding, no net magnetization will refocus due to the influence of the distant dipolar field of the analyte. This problem could be circumvented by either use a gas mixture with a high fraction of xenon [44], or prepolarize a batch of xenon, which is used up only in small fractions at a time.

Another alternative to batch mode would be to polarize Xe inside the magnet [45]. Since Xe and analyte can be kept in different containers, it is possible to admix Rb, which is necessary for optical pumping, to the container with the Xe. The $T_{2}$ of ${ }^{129} \mathrm{Xe}$ can still be very long, even in the presence of $\mathrm{Rb}$ [46], which enables experiments with alternating pumping and probing of Xe magnetization.

From the experiments done in this study, we can conclude that this technique would in principle be feasible in combination with remote detection. Remote detection would allow to use a smaller detection coil with a higher sensitivity, enabling the use of much smaller volumes of xenon gas per detection step. No spectral artifacts would be present due to the slow depletion of the 
xenon magnetization. However, gas mixtures with high percentage of xenon gas would have to be used to minimize the self-diffusion of the sensor. Since configurations are possible where the analyte never gets in contact with the sensor, a setup with circular flow can be set up [47], allowing an economical use of xenon gas, so that even enriched ${ }^{129} \mathrm{Xe}$ could be considered. One problem of a remote experiment is the high vulnerability for multiplicative noise, which requires a very stable magnetic field, thus limiting the applicability with non-superconducting magnets. Other problems might arise due to the strong dependence on a stable flow. One has to wait until the gas has settled after stopping the flow, otherwise the sensitivity is reduced like in the case of high diffusion, prolonging the experiment considerably.

\section{Acknowledgments}

The authors would like to thank Tanya Mazur and Megan Spence, who were involved in an early stage of this project, Janette Ruiz for her help with the polarizer, Sandra Garcia, Lana Chavez, and Song-I Han for the possibility to use their stopped flow setup, and Jamie Walls for insightful discussions. This work was supported by the Director, Office of Science, Office of Basic Energy Sciences, Materials Sciences and Nuclear Science Divisions, of the U.S. Department of Energy under contract DE-AC03-76SF00098. J.G. gratefully acknowledges the Swiss National Science Foundation for support through a postdoctoral fellowship.

\section{References}

[1] B. Goodson, Nuclear magnetic resonance of laser-polarized noble gases in molecules, materials, and organisms, J. Magn. Reson. 155 (2002) 157-216.

[2] S.M. Rubin, M.M. Spence, I.E. Dimitrov, E.J. Ruiz, A. Pines, D.E. Wemmer, Detection of a conformational change in maltose binding protein by Xe-129 NMR spectroscopy, J. Am. Chem. Soc. 123 (2001) 8616-8617.

[3] A. Bifone, Y.-Q. Song, R. Seydoux, R.E. Taylor, B.M. Goodson, T. Pietrass, T.F. Budinger, G. Navon, A. Pines, NMR of laser-polarized xenon in human blood, Proc. Natl. Acad. Sci. USA 93 (1996) 12932-12936.

[4] T. Meersmann, J.W. Logan, R. Simonutti, S. Caldarelli, A. Comotti, P. Sozzani, L.G. Kaiser, A. Pines, Exploring single-file diffusion in one-dimensional nanochannels by laser-polarized Xe-129 NMR spectroscopy, J. Phys. Chem. A 104 (2000) 11665-11670. 
[5] S.M. Rubin, M.M. Spence, A. Pines, D.E. Wemmer, Characterization of the effects of nonspecific xenon-protein interactions on Xe-129 chemical shifts in aqueous solution: Further development of xenon as a biomolecular probe, J. Magn. Reson. 152 (2001) 79-86.

[6] M.M. Spence, S.M. Rubin, I.E. Dimitrov, E.J. Ruiz, D.E. Wemmer, A. Pines, S.Q. Yao, F. Tian, P.G. Schultz, Functionalized xenon as a biosensor, Proc. Natl. Acad. Sci. USA 98 (2001) 10654-10657.

[7] G. Navon, Y.-Q. Song, T. Rõõm, S. Appelt, R.E. Taylor, A. Pines, Enhancement of solution NMR and MRI with laser-polarized xenon, Science, 271 (1996) 18481851.

[8] J.C. Leawoods, B.T. Saam, M.S. Conradi, Polarization transfer using hyperpolarized, supercritical xenon, Chem. Phys. Lett. 327 (2000) 359-364.

[9] M. Luhmer, B.M. Goodson, Y.-Q. Song, D.D. Laws, L. Kaiser, M.C. Cyrier, A. Pines, Study of xenon binding in cryptophane-A using laser-induced NMR polarization enhancement, J. Am. Chem. Soc. 121 (1999) 3502-3512.

[10] T. Brotin, A. Lesage, L. Emsley, A. Collet, Xe-129 NMR spectroscopy of deuterium-labeled cryptophane-A xenon complexes: Investigation of host-guest complexation dynamics, J. Am. Chem. Soc. 122 (2000) 1171-1174.

[11] Y.-Q. Song, B.M. Goodson, R.E. Taylor, D.D. Laws, G. Navon, A. Pines, Selective enhancement of NMR signals for alpha-cyclodextrin with laserpolarized xenon, Angew. Chem. Int. Ed. Engl. 36 (1997) 2368-2370.

[12] T. Rõõm, S. Appelt, R. Seydoux, E.L. Hahn, A. Pines, Enhancement of surface NMR by laser-polarized noble gases, Phys. Rev. B 55 (1997) 11604-11610.

[13] A.J. Moulé, M.M. Spence, S. Han, J.A. Seeley, K.L. Pierce, S.K. Saxena, A. Pines, Amplification of xenon NMR and MRI by remote detection, Proc. Natl. Acad. Sci. USA 100 (2003) 9122-9127.

[14] J.A. Seeley, S. Han, A. Pines, Remotely detected high-field MRI of porous samples, J. Magn. Reson. 167 (2004) 282-290.

[15] W.S. Warren, W. Richter, A.H. Andreotti, B.T. Farmer, Generation of impossible cross-peaks between bulk water and biomolecules in solution NMR, Science 262 (1993) 2005-2009.

[16] W. Richter, S.H. Lee, W.S. Warren, Q.H. He, Imaging with intermolecular multiple-quantum coherences in solution nuclear magnetic resonance, Science 267 (1995) 654-657.

[17] R. Bowtell, Indirect detection via the dipolar demagnetizing field, J. Magn. Reson. 100 (1992) 1-17.

[18] M.P. Augustine, K.W. Zilm, Observation of bulk susceptibility effects in highresolution nuclear magnetic resonance, J. Magn. Reson. A 123 (1996) 145-156. 
[19] K.W. Zilm, Optically pumped NMR in high magnetic fields, American Physical Society Meeting, Pittsburgh PA, USA (1994).

[20] W.S. Warren, S. Ahn, The boundary between liquidlike and solidlike behavior in magnetic resonance, J. Chem. Phys. 108 (1998) 1313-1325.

[21] P.R. Bachiller, S. Ahn, W.S. Warren, Detection of intermolecular heteronuclear multiple-quantum coherences in solution NMR, J. Magn. Reson. A 122 (1996) 94-99.

[22] M.P. Augustine, PhD Thesis, Yale (1995).

[23] A.S. Verhulst, O. Liivak, M.H. Sherwood, I.L. Chuang, A rapid and precise probe for measurement of liquid xenon polarization, J. Magn. Reson. 155 (2002) $145-149$.

[24] I.E. Dimitrov, E. Insko, R. Rizi, J.S. Leigh, Indirect detection of lung perfusion using susceptibility-based hyperpolarized gas imaging, J. Magn. Reson. Imag. 21 (2005) 149-155.

[25] S. Lee, W. Richter, S. Vathyam, W.S. Warren, Quantum treatment of the effects of dipole-dipole interactions in liquid nuclear magnetic resonance, J. Chem. Phys. 105 (1996) 874-900.

[26] S. Huang, Y.-Y. Lin, N. Lisitza, W.S. Warren, Signal interferences from turbulent spin dynamics in solution nuclear magnetic resonance spectroscopy, J. Chem. Phys. 116 (2002) 10325-10337.

[27] A.L. Zook, B.B. Adhyaru, C.R. Bowers, High capacity production of $>65 \%$ spin polarized xenon-129 for NMR spectroscopy and imaging, J. Magn. Reson. 159 (2002) 175-182.

[28] R.R. Ernst, G. Bodenhausen, A. Wokaun, Principles of Nuclear Magnetic Resonance in One and Two Dimensions, Clarendon Press, Oxford, 1987.

[29] W.S. Warren, S. Lee, W. Richter, S. Vathyam, Correcting the classical dipolar demagnetizing field in solution NMR, Chem. Phys. Lett. 247 (1995) 207-214.

[30] W. Richter, W.S. Warren, Intermolecular multiple quantum coherences in liquids, Concepts Magn. Reson. 12 (2000) 396-409.

[31] N. Murali, A. Kumar, Multiple-quantum artifacts in single-quantum twodimensional correlated NMR spectra of strongly coupled spins, Chem. Phys. Lett. 128 (1986) 58-61.

[32] N. Bloembergen, R. Pound, Radiation damping in magnetic resonance experiments, Phys. Rev. 95 (1954) 8-12.

[33] S.Y. Huang, J.D. Walls, Y. Wang, W.S. Warren, Y.-Y. Lin, Signal irreproducibility in high-field solution magnetic resonance experiments caused by spin turbulence, J. Chem. Phys. 121 (2004) 6105-6109.

[34] A.F. Mehlkopf, D. Korbee, T.A. Tiggelman, R. Freeman, Sources of $t_{1}$ noise in two-dimensional NMR, J. Magn. Reson. 58 (1984) 315-323. 
[35] J. Granwehr, Multiplicative noise in NMR spectroscopy, Concepts Magn. Reson. (submitted).

[36] J.M. Nuzillard, R. Freeman, Oversampling in two-dimensional NMR, J. Magn. Reson. A 110 (1994) 252-256.

[37] L. Zhao, R. Mulkern, C.-H. Tseng, D. Williamson, S. Patz, R. Kraft, R.L. Walsworth, F.A. Jolesz, M.S. Albert, Gradient-echo imaging considerations for hyperpolarized ${ }^{129}$ Xe MR, J. Magn. Reson. B 113 (1996) 179-183.

[38] J.T. Urban, PhD Thesis, University of California at Berkeley (2005).

[39] H.T. Edzes, The nuclear magnetization as the origin of transient changes in the magnetic field in pulsed NMR experiments, J. Magn. Reson. 86 (1990) 293-303.

[40] C.J. Jameson, K. Jameson, S.M. Cohen, Temperature and density dependence of ${ }^{129}$ Xe chemical shift in xenon gas, J. Chem. Phys. 59 (1973) 4540-4546.

[41] R.C. Weast, Handbook of Chemistry and Physics, 54th ed., CRC Press, Cleveland, 1973.

[42] G. Bodenhausen, H. Kogler, R.R. Ernst, Selection of coherence-transfer pathways in NMR pulse experiments, J. Magn. Reson. 58 (1984) 370-388.

[43] S. Månsson, E. Johansson, J. Svensson, L.E. Olsson, F. Ståhlberg, J.S. Petersson, K. Golman, Echo-planar MR imaging of dissolved hyperpolarized ${ }^{129}$ Xe, Acta Radiologica 43 (2002) 455-460.

[44] R. Wang, R.W. Mair, M.S. Rosen, D.G. Cory, R.L. Walsworth, Simultaneous measurement of rock permeability and effective porosity using laser-polarized noble gas NMR, Phys. Rev. E 70 (2004) 026312.

[45] M.P. Augustine, K.W. Zilm, Optical pumping magnetic resonance in high magnetic fields: Characterization of nuclear relaxation during pumping, J. Chem. Phys. 105 (1996) 2998-3011.

[46] D. Raftery, H.W. Long, D. Shykind, P.J. Grandinetti, A. Pines. Multiple-pulse nuclear magnetic resonance of optically pumped xenon in a low magnetic field, Phys. Rev. A 50 (1994) 567-574.

[47] K. Knagge, J. Prange, D. Raftery D, A continuously recirculating optical pumping apparatus for high xenon polarization and surface NMR studies, Chem. Phys. Lett. 397 (2004) 11-16. 